\title{
Rapidly Destructive Coxopathy: an orthopedic and radiological challenge
}

\author{
Akira Toga ${ }^{1}$, Ayush Balaji ${ }^{2}$, and Atsushi Funayama ${ }^{1}$ \\ ${ }^{1}$ Saiseikai Yokohamashi Tobu Hospital \\ ${ }^{2}$ Edogawa Hospital
}

June 28, 2021

\begin{abstract}
Rapidly Destructive Coxopathy is a rare orthopedic manifestation that results in the damage of the hip joint in a very limited timespan causing severe hip pain and disability. It is difficult to differentiate from osteoarthritis upon initial examination and can only be distinguished through frequent follow-ups and radiographic evaluation.
\end{abstract}

\section{Key Clinical Message}

The rapid progression of RDC can cause challenges when surgery is delayed therefore clinicians have limited time to act before the complete destruction of the joint. Acting early will improve operative outcomes and quality-of-life.

Keywords: Joint conditions, Total hip arthroplasty; Osteoarthritis;
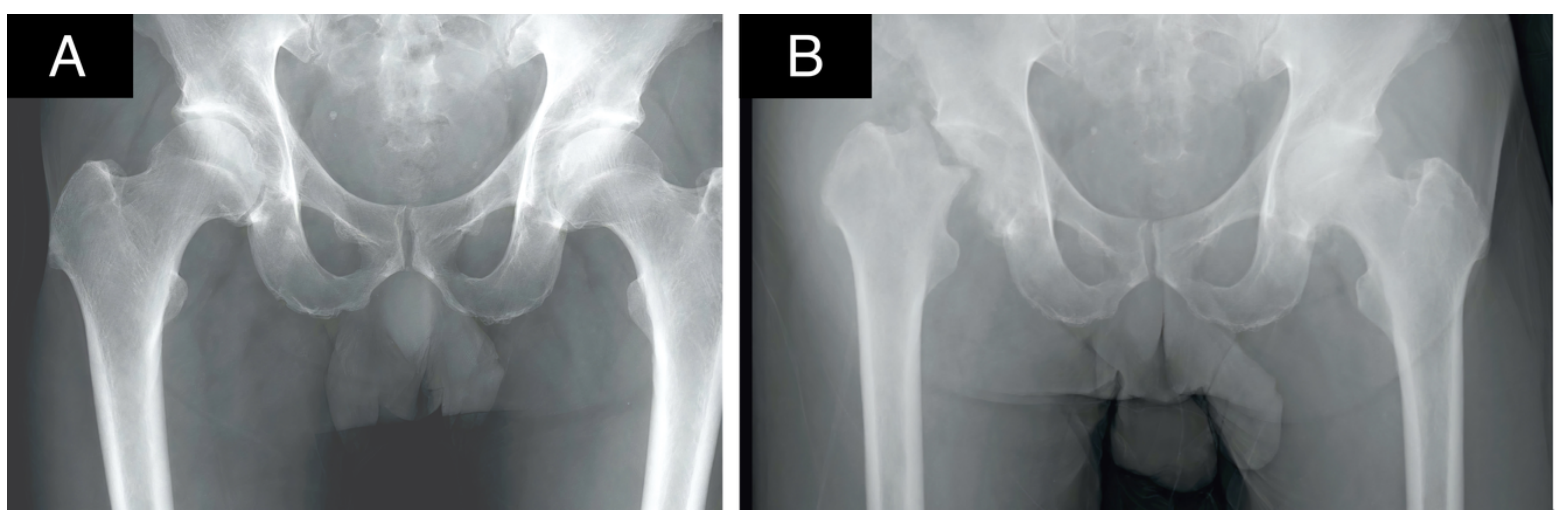

Figure 1: (A) Pre-operative radiograph at first visit showing eburnation of the right hip joint. (B) Radiograph at two-weeks follow up visit, prior to the total hip arthroplasty (THA), showing the complete destruction of the femoral head and erosion of the acetabulum, there is also a visible reduction in the left hip joint space.

\section{Case}

A 62-year-old man with no significant medical history presented with right hip pain. Physical examination revealed increased right hip pain with rotation and flexion and radiographic evaluation indicated decreased 
joint space with cartilage destruction. The patient revealed that he felt increasing pain in his right hip 3 months before the first visit and required a cane to walk. 2 milliliters of synovial fluid was withdrawn to eliminate bacterial infection. 2 weeks later, the patient needed support for weight-bearing and his femoral head had significantly deteriorated. Radiographic study revealed rapid destruction of the femoral head which is a characteristic of Rapidly Destructive Coxopathy (RDC). Other possible markers include subchondral insufficiency which can be visible on magnetic resonance imaging (MRI), destruction of articular cartilage, and secondary osseous differences. ${ }^{1}$ The main characteristic of RDC is micro-fracture which distinguishes it from osteoarthritis, and other related pathologies. ${ }^{2}$ Subsequently, the signs of RDC was visible in his left hip during follow-up visits. The rapid progression of the condition can cause challenges when surgery is delayed therefore clinicians have limited time to act before the complete destruction of the joint. Acting early will

improve operative outcomes and quality-of-life.

\section{Disclosure}

The authors declare no potential conflicts of interest.

\section{Funding Statement}

The authors received no financial support for the research, authorship, and/or publication of this article.

\section{Informed Consent}

Informed consent was obtained from the patient for publication.

\section{Data Availability}

Data sharing not applicable to this article as no datasets were generated or analyzed during the current study.

\section{Author Contributions}

Akira Toga: contributed to write-up of abstract, case presentation, and images

Ayush Balaji: contributed to write-up of abstract, case presentation, and images

Atsushi Funayama: reviewed and edited the manuscript.

\section{References}

Watanabe W, Itoi E, Yamada S. Early MRI findings of rapidly destructive coxarthrosis. Skeletal Radiol . 2002;31(1):35-38.

Batra S, Batra M, McMurtrie A, Sinha AK. Rapidly destructive osteoarthritis of the hip joint: a case series. J Orthop Surg Res . 2008;3:3. 